\title{
Neutron diffraction study of average and local structure in $\mathrm{La}_{0.5} \mathrm{Ca}_{0.5} \mathrm{MnO}_{3}$
}

\author{
E. E. Rodriguez, Th. Proffen $*$ A. Llobet, and J. J. Rhyne \\ J. F. Mitchell \\ Argonne National Laboratory, Argonne, IL
}

Lujan Neutron Scattering Center, Los Alamos National Laboratory,Los Alamos, NM.

(Dated: July 13, 2018)

\begin{abstract}
We used neutron powder diffraction to obtain the local and long-range structure of $\mathrm{La}_{0.5} \mathrm{Ca}_{0.5} \mathrm{MnO}_{3}$ at room temperature and $20 \mathrm{~K}$. By combining Rietveld and pair distribution function analysis of the total neutron scattering data, we have analyzed the structure of the compound using two competing models describing the low temperature phase: first the charge ordered/orbital ordered model and second the Mn-Mn dimer model. These structural models fit the $20 \mathrm{~K}$ neutron powder diffraction pattern equally well using Rietveld analysis. Therefore, pair distribution function analysis is used to probe the local and medium-range structure revealing a system with two distinctly distorted Mn octahedra and Mn ions with non-integral valence states. The distorted octahedra differ with the structural model for the Zener polaron type Mn-Mn dimer picture proposed for $\operatorname{Pr}_{0.60} \mathrm{Ca}_{0.40} \mathrm{MnO}_{3}$ and order in a similar checkerboard configuration associated with the CE-type anti-ferromagnetic structure. Therefore, locally the charge difference and structural ordering between the two $\mathrm{Mn}$ is appreciable enough to describe the system at $20 \mathrm{~K}$ as "partially charge ordered".
\end{abstract}

\section{INTRODUCTION}

Understanding the relation between the magnetic ordering, electronic ground state, and crystallographic structure in mixed valence manganites has preoccupied experimentalists and theorists for over 50 years. Today, manganites continue to be an interesting topic in solidstate physics because of their high degree of functionality, strongly correlated electrons, and ability to exhibit fascinating physical phenomena such as colossal magnetoresistance (CMR) $\stackrel{1}{\underline{1}}$ In 1955 Wollan and Koeler completed their classic neutron powder diffraction (NPD) study of the effects of electronic doping on magnetic, structural, and transport properties of the manganese perovskite $\mathrm{La}_{1-x} \mathrm{Ca}_{x} \mathrm{MnO}_{3}{ }_{2}^{2}$. Since $\mathrm{LaMnO}_{3}$ and $\mathrm{CaMnO}_{3}$ have $\mathrm{Mn}$ with formal valences of III ( $\mathrm{d}^{4}$ configuration) and IV $\left(\mathrm{d}^{3}\right.$ configuration) respectively, the system is hole doped as $\mathrm{La}^{3+}$ is substituted by $\mathrm{Ca}^{2+}$. The half-doped compound is an especially interesting composition to study because it is on a metal to insulator transition interface in the phase diagram. Moreover, one would expect a ratio of $\mathrm{Mn}^{4+} / \mathrm{Mn}^{3+}=1$ due to an equal amount of divalent (Ca) and trivalent $(\mathrm{La})$ cations. ${ }^{2}$

A qualitative interpretation of Wollan and Koeler's results proposed by Goodenough predicted that at low temperatures the $\mathrm{Mn}^{3+}$ and $\mathrm{Mn}^{4+}$ ions in the $x=0.5$ compound would order in alternate (110) planes. $\frac{3}{\underline{\underline{3}}}$ The structure resulting from $\mathrm{Mn}^{4+} / \mathrm{Mn}^{3+}$ ordering has been termed the charge-ordered (CO) structure and its associated magnetic ordering is known as the CE-type antiferromagnetic (AFM) structure. Moreover, the $\mathrm{Mn}^{3+}$ octahedra are Jahn-Teller distorted and consequently the $\mathrm{d}_{z}^{2}$ orbitals order in a zigzag pattern; this is described as orbital ordering (OO) and is coupled with $\mathrm{CO}$ in Goodenough's model. Since the $\mathrm{Mn}^{4+}$ octahedra do not distort, their positions are modulated throughout the structure with an amplitude that is commensurate with the crystal lattice.

Since the 1990's, Goodenough's interpretation of the charge-ordered state has been revised and challenged by new experimental studies. The neutron and x-ray powder diffraction study by Radaelli et al $\underline{\underline{4}}$ suggest that the modulation of the $\mathrm{Mn}^{4+}$ octahedra quasicommensurate at low temperature and still confirms Goodenough's predictions of charge/orbital ordering $(\mathrm{CO} / \mathrm{OO}) \stackrel{4}{*}$ However, the $\mathrm{CO} / \mathrm{OO}$ picture has been challenged by $\mathrm{x}$-ray resonant scattering (RXD), x-ray absorption near-edge spectroscopy (XANES) and single-crystal neutron diffraction studies $5 \cdot 6 \cdot 7.8 .9 .10$ These studies conclude that the local structure around the $\mathrm{Mn}$ atoms is incompatible with Goodenough's Mn-O bonding model and that the Mn atoms do not have integral valence states. In addition, one XANES study found that one of the Mn octahedra is Jahn-Teller distorted while the other Mn octahedron lost their local tetrahedral point group symmetry $\frac{5}{\underline{5}}$ In short, the study concludes that both octahedra are distorted but in a different manner making the ionic picture of $\mathrm{CO} / \mathrm{OO}$ unrealistic.

Meanwhile, the study by Daoud-Aladine and Rodriguez-Carvajal suggests that the $\mathrm{CO} / \mathrm{OO}$ model is inconsistent with single crystal diffraction data of $\operatorname{Pr}_{0.6} \mathrm{Ca}_{0.4} \mathrm{MnO}_{3}$, a system similar to $\mathrm{La}_{1-x} \mathrm{Ca}_{x} \mathrm{MnO}_{3} \stackrel{8,9,10}{=}$ Daoud-Aladine reinterpreted the complex magnetic ordering and the modulated structural distortion as a consequence of ferromagnetic (FM) MnMn dimer ordering. In this model the formal valences of the $\mathrm{Mn}$ are only fractional $\left(\mathrm{Mn}^{+3.5+\delta} / \mathrm{Mn}^{+3.5-\delta}\right)$ and all the octahedra are elongated similarly so that two octahedra connect along their elongated direction. ${ }^{3}$ The FM Mn-Mn dimer in this model is likened to a Zener polaron $\underline{\underline{8}}$ A Zener polaron is defined as a pair of $\mathrm{Mn}^{3+}$ cations that share a hole on a bridging oxygen 
and in which a Zener double exchange mechanism is responsible for the FM coupling of the Mn atoms 11.12 It is important to note that orbital ordering also occurs within the Mn-Mn dimer model.

Our study aims to use long-range and short-range analysis of neutron powder diffraction data to provide further insight into the modulated distortions seen at lower temperatures. Rietveld analysis alone will only yield the average structure of the material. Here we combine Rietveld analysis with a local structure analysis technique called the pair distribution function (PDF). The PDF is the Fourier transform of the total scattering pattern containing both Bragg as well as diffuse scattering. This approach will result in short, medium and long range structural information, depending on the refinement range $\mathrm{r}_{\max }$. The PDF approach has been used extensively to study the local structure of other manganites 13.14 .15 .16 .17 and a great overview of the technique applied to a variety of complex materials can be found here 18

\section{EXPERIMENTAL}

Samples were prepared by conventional ceramic methods. Before weighing, $\mathrm{La}_{2} \mathrm{O}_{3}(99.99 \%)$ was fired at $1000{ }^{\circ} \mathrm{C}$ in $\mathrm{O}_{2}, \mathrm{MnO}_{2}(99.999 \%)$ was treated in flowing oxygen at $425^{\circ} \mathrm{C}$ then slow-cooled; $\mathrm{CaCO} 3$ was dried at $100{ }^{\circ} \mathrm{C}$. Stoichiometric quantities were mixed and calcined at $900{ }^{\circ} \mathrm{C}$, then sintered between $1100{ }^{\circ} \mathrm{C}$ and $1380{ }^{\circ} \mathrm{C}$ with intermediate grinding and pelletizing. The final pellet was annealed in $\mathrm{O}_{2}$ and slow cooled to ensure stoichiometry.

Neutron powder diffraction data were collected on $\mathrm{NPDF}^{19}$ and HIPD neutron diffractometers located at the Lujan Neutron Scattering Center at Los Alamos National Laboratory. The sample of about $10 \mathrm{~g}$ was sealed in a cylindrical vanadium tube with helium exchange gas. Data were collected at temperatures between $\mathrm{T}=300 \mathrm{~K}$ down to $\mathrm{T}=20 \mathrm{~K}$ in a closed cycle helium refrigerator. For PDF analysis the data are corrected for detector deadtime and efficiency, background, absorption, multiple scattering, inelasticity effects and normalized by the incident flux and the total sample scattering cross-section to yield the total scattering structure function, $S(Q)$. This is Fourier transformed according to

$$
G(r)=\frac{2}{\pi} \int_{0}^{\infty} Q[S(Q)-1] \sin (Q r) d Q
$$

The data were terminated at a value of $\mathrm{Q}_{\max }=35 \AA^{-1}$. Data processing was carried out using the program PDFgetN ${ }^{20}$ Data collection and analysis procedures have been described elsewhere. 18
TABLE I: RT model used in Rietveld analysis of $300 \mathrm{~K}$ data. Orthorhombic lattice with Pbnm symmetry. Lattice constants $a=5.4309(1) \AA, b=5.4211(1) \AA$, and $c=7.6400(1) \AA$. $\mathrm{R}_{\mathrm{wp}}=4.4 \%$ and $\chi^{2}=6.2$.

\begin{tabular}{lcccc}
\hline \hline Atom & Wyck pos. & $x$ & $y$ & $z$ \\
\hline Mn & $4 \mathrm{~b}$ & 0.5 & 0.0 & 0.0 \\
La/Ca & $4 \mathrm{c}$ & $-0.0036(2)$ & $0.0195(1)$ & 0.25 \\
O1 & $4 \mathrm{c}$ & $0.0599(1)$ & $0.4918(2)$ & 0.25 \\
$\mathrm{O} 2$ & 8d & $0.7236(1)$ & $0.2770(1)$ & $0.0312(1)$ \\
\hline \hline
\end{tabular}
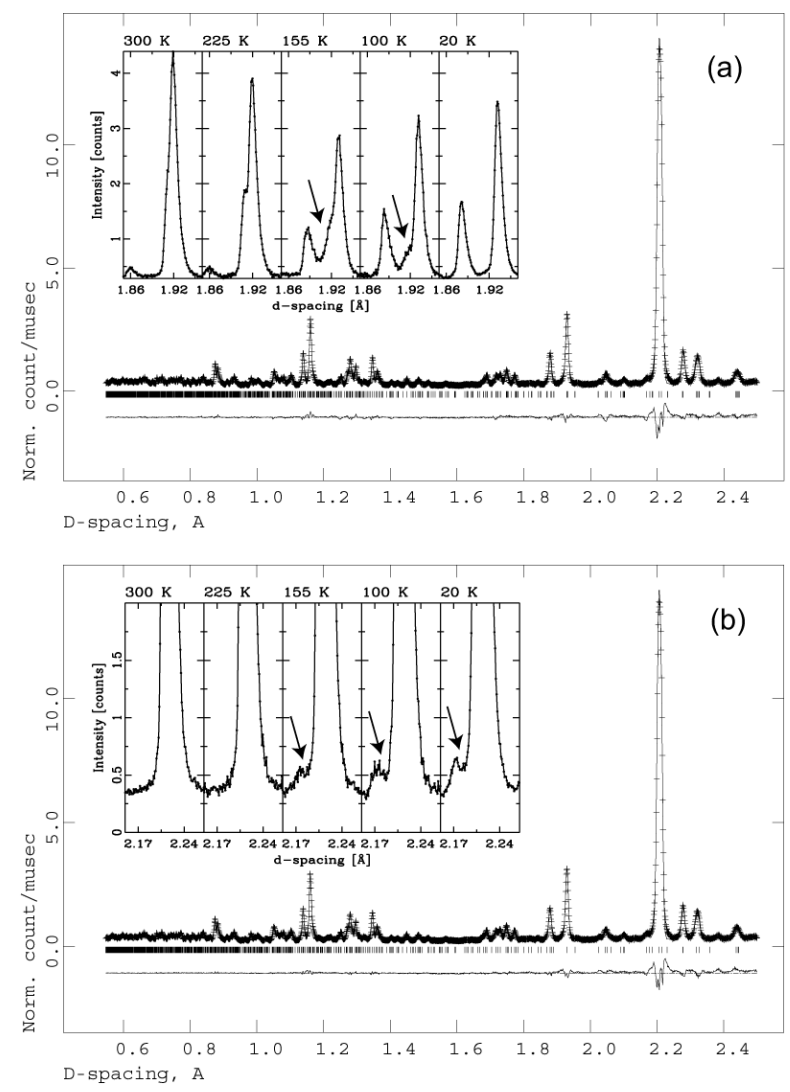

FIG. 1: (a) NPD pattern at $20 \mathrm{~K}$ fitted with the LT-M model. Arrows in inset indicate contribution from the room temperature phase. (b) The LT-O model fit of the $20 \mathrm{~K}$ data. Arrows in inset indicate the super-lattice peaks. The bottom line below the tick marks is the difference between the observed and calculated NPD pattern. The temperature evolution in the insets are NPD patterns measured from HIPD and the fits are done for NPDF data.

\section{RESULTS AND ANALYSIS}

\section{A. Rietveld Analysis}

The Rietveld refinements were carried out using the GSAS Rietveld code. ${ }^{21}$ At room temperature the structure of $\mathrm{La}_{0.5} \mathrm{Ca}_{0.5} \mathrm{MnO}_{3}$ is single phase and orthorhombic with space group Pbnm. The parameters of the room temperature phase, denoted RT, are listed in Table 1 At $\mathrm{T}=20 \mathrm{~K}$ only one phase was observed and found to have 
TABLE II: LT-M model used in Rietveld analysis of $20 \mathrm{~K}$ data with the values of $\Delta x, \Delta x^{\prime}$, and the position of atoms refined with $P 2_{1} / m$ symmetry constraints. The monoclinic cell is based on an orthorhombic cell doubled in the $b$ direction. The $y$ and $z$ coordinates for $\mathrm{Mn}$ and $\mathrm{O} 2$ atoms are fixed. $\Delta x=0.0220(1), \Delta x^{\prime}=0.0103(3), a=5.47450(7) \AA, b=$ $10.88703(13) \AA, c=7.51880(8) \AA, \gamma=89.967(3)^{\circ} . \mathrm{R}_{\mathrm{wp}}=5.7 \%$ and $\chi^{2}=10.2$.

\begin{tabular}{lcccc}
\hline \hline Atom & Wyck pos. & $x$ & $y$ & $z$ \\
\hline $\mathrm{Mn}(1)$ & $2 \mathrm{a}$ & 0.0 & 0.0 & 0.0 \\
$\mathrm{Mn}(2)$ & $2 \mathrm{~b}$ & 0.0 & 0.5 & 0.0 \\
$\mathrm{Mn}(3)$ & 4f & $0.5-\Delta x$ & 0.25 & 0.0 \\
$\mathrm{La}(1)$ & $2 \mathrm{e}$ & $0.4990(5)$ & $0.0117(4)$ & 0.25 \\
$\mathrm{La}(2)$ & $2 \mathrm{e}$ & $0.4952(5)$ & $0.5068(4)$ & 0.25 \\
$\mathrm{La}(3)$ & $2 \mathrm{e}$ & $0.0057-\Delta x^{\prime}$ & $0.2601(4)$ & 0.25 \\
$\mathrm{La}(4)$ & $2 \mathrm{e}$ & $0.0057+\Delta x^{\prime}$ & $0.7569(3)$ & 0.25 \\
$\mathrm{O} 1(1)$ & $2 \mathrm{e}$ & $-0.0592(6)$ & $-0.0133(3)$ & 0.25 \\
$\mathrm{O} 1(2)$ & $2 \mathrm{e}$ & $-0.0538(5)$ & $0.4980(3)$ & 0.25 \\
$\mathrm{O} 1(3)$ & $2 \mathrm{e}$ & $0.5660-\Delta x$ & $0.2532(4)$ & 0.25 \\
$\mathrm{O} 1(4)$ & $2 \mathrm{e}$ & $0.5660+\Delta x$ & $0.7470(4)$ & 0.25 \\
$\mathrm{O} 2(1)$ & 4f & $0.2248-\Delta x$ & 0.1440 & 0.0337 \\
$\mathrm{O} 2(2)$ & 4f & $0.7198-\Delta x$ & 0.1151 & -0.0337 \\
$\mathrm{O} 2(3)$ & 4f & $0.2248+\Delta x$ & 0.6440 & 0.0337 \\
$\mathrm{O} 2(4)$ & 4f & $0.7198+\Delta x$ & 0.6151 & -0.0337 \\
\hline \hline
\end{tabular}

TABLE III: Model LT-M' used in Rietveld analysis of $20 \mathrm{~K}$ data. Atom positions refined with $P 2_{1} / m$ symmetry constraints. $a=5.4750(51) \AA, b=10.8885(1) \AA, c=7.5194(1) \AA$, $\gamma=89.950(1)^{\circ} . R_{\mathrm{wp}}=4.5 \%$ and $\chi^{2}=6.5$.

\begin{tabular}{lcccc}
\hline \hline Atom & Wyck pos. & $x$ & $y$ & $z$ \\
\hline $\mathrm{Mn}(1)$ & $2 \mathrm{a}$ & 0.0 & 0.0 & 0.0 \\
$\mathrm{Mn}(2)$ & $2 \mathrm{~b}$ & 0.0 & 0.5 & 0.0 \\
$\mathrm{Mn}(3)$ & $4 \mathrm{f}$ & $0.5157(4)$ & $0.2469(3)$ & $0.0078(3)$ \\
$\mathrm{La}(1)$ & $2 \mathrm{e}$ & $0.0234(5)$ & $0.2589(3)$ & 0.25 \\
$\mathrm{La}(2)$ & $2 \mathrm{e}$ & $-0.0090(5)$ & $0.7644(4)$ & 0.25 \\
$\mathrm{La}(3)$ & $2 \mathrm{e}$ & $0.5018(6)$ & $0.0051(3)$ & 0.25 \\
$\mathrm{La}(4)$ & $2 \mathrm{e}$ & $0.4924(5)$ & $0.5121(4)$ & 0.25 \\
$\mathrm{O} 1(1)$ & $2 \mathrm{e}$ & $-0.0577(6)$ & $0.0003(4)$ & 0.25 \\
$\mathrm{O} 1(2)$ & $2 \mathrm{e}$ & $-0.0662(5)$ & $0.4890(3)$ & 0.25 \\
$\mathrm{O} 1(3)$ & $2 \mathrm{e}$ & $0.5913(4)$ & $0.2472(2)$ & 0.25 \\
$\mathrm{O} 1(4)$ & $2 \mathrm{e}$ & $0.5483(5)$ & $0.7470(4)$ & 0.25 \\
$\mathrm{O} 2(1)$ & $4 \mathrm{f}$ & $0.2577(4)$ & $0.1360(3)$ & $0.0345(4)$ \\
$\mathrm{O} 2(2)$ & $4 \mathrm{f}$ & $0.2069(4)$ & $0.6355(3)$ & $0.0352(4)$ \\
$\mathrm{O} 2(3)$ & $4 \mathrm{f}$ & $0.7372(3)$ & $0.1129(4)$ & $-0.0366(4)$ \\
$\mathrm{O} 2(4)$ & $4 \mathrm{f}$ & $0.7073(4)$ & $0.6132(2)$ & $-0.0294(4)$ \\
\hline \hline
\end{tabular}

antiferromagnetic ordering of the CE-type. In between $225 \mathrm{~K}$ and $100 \mathrm{~K}$ there is a mixture of the RT and LT phases as shown in Fig. 17 inset. As the temperature is lowered, the low temperature phase develops within the room temperature phase and its unit cell anisotropically distorts. This is similar to other NPD and XRD studies noting coexisting phases from around $210 \mathrm{~K}$ down to low temperatures ${ }^{4.22}$ In addition, the room temperature phase develops long-range ferromagnetism at $\mathrm{T}_{c}=195 \mathrm{~K}$ and the low temperature phase undergoes an AFM ordering transition at $\mathrm{T}_{N}=155 \mathrm{~K}$. Unlike other NPD studies
TABLE IV: Model LT-O used in Rietveld analysis of $20 \mathrm{~K}$ data. Atom positions refined with $P 2_{1} n m$ symmetry constraints. $a=5.47507(5) \AA, b=10.88833(8) \AA, c=7.5193(1) \AA$. $\mathrm{R}_{\mathrm{wp}}=4.4 \%$ and $\chi^{2}=6.2$.

\begin{tabular}{lcccc}
\hline \hline Atom & Wyck pos. & $x$ & $y$ & $z$ \\
\hline $\mathrm{Mn}(1)$ & $4 \mathrm{~b}$ & $0.0176(8)$ & $0.3742(4)$ & $0.2537(6)$ \\
$\mathrm{Mn}(2)$ & $4 \mathrm{~b}$ & $0.9923(11)$ & $0.8764(4)$ & $0.2445(6)$ \\
$\mathrm{La}(1)$ & $2 \mathrm{a}$ & $0.5017(8)$ & $0.3603(3)$ & 0.0 \\
$\mathrm{La}(2)$ & $2 \mathrm{a}$ & $0.5244(8)$ & $0.3838(4)$ & 0.5 \\
$\mathrm{La}(3)$ & $2 \mathrm{a}$ & $0.4923(8)$ & $0.8676(3)$ & 0.0 \\
$\mathrm{La}(4)$ & $2 \mathrm{a}$ & $0.4906(8)$ & $0.8827(3)$ & 0.5 \\
$\mathrm{O} 1(1)$ & $2 \mathrm{a}$ & $0.0768(10)$ & $0.3692(5)$ & 0.5 \\
$\mathrm{O} 1(2)$ & $2 \mathrm{a}$ & $0.9448(7)$ & $0.3815(4)$ & 0.0 \\
$\mathrm{O} 1(3)$ & $2 \mathrm{a}$ & $0.0503(7)$ & $0.8811(3)$ & 0.5 \\
$\mathrm{O} 1(4)$ & $2 \mathrm{a}$ & $0.9187(9)$ & $0.8852(4)$ & 0.0 \\
$\mathrm{O} 2(1)$ & $4 \mathrm{~b}$ & $0.8020(5)$ & $0.5108(2)$ & $0.2817(4)$ \\
$\mathrm{O} 2(2)$ & $4 \mathrm{~b}$ & $0.7265(0)$ & $0.7605(2)$ & $0.2885(4)$ \\
$\mathrm{O} 2(3)$ & $4 \mathrm{~b}$ & $0.2289(10)$ & $0.7364(3)$ & $0.2176(5)$ \\
$\mathrm{O} 2(4)$ & $4 \mathrm{~b}$ & $0.7482(5)$ & $0.0104(2)$ & $0.2846(3)$ \\
\hline \hline
\end{tabular}

our data does not show any contribution from the remnant room temperature phase at $20 \mathrm{~K}$ (Fig. 10 inset).

Small satellite peaks observed at $155 \mathrm{~K}$ are indicative of the orbital ordering transition and cannot be fit with model describing the room temperature phase, denoted model RT. (Fig. 1b). The most intense satellite peak at $q=[1,1 / 2,1]$ is created by the modulated displacement of the Mn octahedra. Three structural models were used to fit the satellite peaks in the $20 \mathrm{~K}$ data. The first model, denoted model LT-M, is the structural model describing $\mathrm{CO} / \mathrm{OO}$ at low temperatures as defined by Radaelli et al $\stackrel{4}{n}$ In model LT-M the monoclinic lattice is doubled in the b-direction and the bond lengths around the $\mathrm{Mn}^{4+}$ are fixed to be approximately $1.915 \AA$. Two modulation amplitudes $\Delta x$ and $\Delta x^{\prime}$ are applied to the positions of the equatorial $\mathrm{O}, \mathrm{Mn}^{4+}$, and half of the $\mathrm{La} / \mathrm{Ca}$ atoms as shown in Table III Along with $\Delta x$ and $\Delta x^{\prime}$, the remainder of the atoms' positions are refined with $P 2_{1} / m$ symmetry constraints. The goodness of fit parameters obtained were a $\chi^{2}$ of 10.2 and an $\mathrm{R}_{\mathrm{wp}}$ of $5.7 \%$.

However, if these constraints are loosened to allow atomic positions to adjust freely within the symmetry allowed by the space group $P 2_{1} / m$ but with no additional constraints on the $\mathrm{Mn}^{4+}-\mathrm{O}$ bonds, the $\mathrm{R}_{\mathrm{wp}}$ and $\chi^{2}$ values improve to values of $4.5 \%$ and 6.5 respectively. This model, denoted LT-M', is less constrained than model LT-M because it does not fix the $\mathrm{Mn}^{4+}-\mathrm{O}$ bond and does not refine modulation vectors $\Delta x$ and $\Delta x^{\prime}$. Although the goodness of fit parameters improve in model LT-M' one should note, that the number of parameters refined in the non-linear least squares fit are twice the number as those refined in the model LT-M. The parameters of model LTM' are listed in Table $\amalg$ and the Mn-O bond lengths are listed in Table $\nabla$ The LT-M' model has many similarities with the LT-M model except for the distribution of $\mathrm{Mn}^{4+}-\mathrm{O}$ bond distances. The resulting bond distances give some indication that the $\mathrm{Mn}^{4+}$ octahedra may not 
TABLE V: Structural information from Rietveld analysis of models RT, LT-M, LT-M', and LT-O. The angles and lengths are concerned only with the Mn to equatorial O bonds. Only model RT is used for $300 \mathrm{~K}$ data, the rest for $20 \mathrm{~K}$ data.

\begin{tabular}{|c|c|c|c|c|}
\hline \multirow{3}{*}{$\begin{array}{l}\text { Model } \\
\text { RT }\end{array}$} & \multicolumn{2}{|c|}{ Mn- $\mathrm{O}_{e q}$ bond distances $(\AA)$} & \multicolumn{2}{|c|}{$\mathrm{Mn}-\mathrm{O}_{e q}$ bond angles $\left({ }^{\circ}\right)$} \\
\hline & $\mathrm{Mn}-\mathrm{O}$ & $2 \times 1.94562(1)$ & $\mathrm{Mn}-\mathrm{O}-\mathrm{Mn}$ & $161.3861(1)$ \\
\hline & $\mathrm{Mn}-\mathrm{O}$ & $2 \times 1.94321(1)$ & & \\
\hline \multirow[t]{6}{*}{ LT-M } & $\mathrm{Mn}^{3+}(1)-\mathrm{O}$ & $2 \times 1.93789(6)$ & $\mathrm{Mn}^{3+}(1)-\mathrm{O}-\mathrm{Mn}^{4+}$ & $157.130(1)$ \\
\hline & $\mathrm{Mn}^{3+}(1)-\mathrm{O}$ & $2 \times 2.09058(6)$ & & $160.1521(9)$ \\
\hline & $\mathrm{Mn}^{3+}(2)-\mathrm{O}$ & $2 \times 1.90579(6)$ & $\mathrm{Mn}^{3+}(2)-\mathrm{O}-\mathrm{Mn}^{4+}$ & $161.2382(8)$ \\
\hline & $\mathrm{Mn}^{3+}(2)-\mathrm{O}$ & $2 \times 2.08533(6)$ & & $162.2580(7)$ \\
\hline & $\mathrm{Mn}^{4+}-\mathrm{O}$ & $2 \times 1.91551(6)$ & & \\
\hline & $\mathrm{Mn}^{4+}-\mathrm{O}$ & $2 \times 1.91464(6)$ & & \\
\hline \multirow[t]{8}{*}{$\overline{\text { LT-M' }}$} & $\mathrm{Mn}^{3+}(1)-\mathrm{O}$ & $2 \times 1.91209(5)$ & $\mathrm{Mn}^{3+}(1)-\mathrm{O}-\mathrm{Mn}^{4+}$ & $158.7380(7)$ \\
\hline & $\mathrm{Mn}^{3+}(1)-\mathrm{O}$ & $2 \times 2.06250(5)$ & & $165.4079(4)$ \\
\hline & $\mathrm{Mn}^{3+}(2)-\mathrm{O}$ & $2 \times 1.87984(5)$ & $\mathrm{Mn}^{3+}(2)-\mathrm{O}-\mathrm{Mn}^{4+}$ & $159.3062(6)$ \\
\hline & $\mathrm{Mn}^{3+}(2)-\mathrm{O}$ & $2 \times 2.03272(5)$ & & $162.4260(7)$ \\
\hline & $\mathrm{Mn}^{4+}-\mathrm{O}$ & $1.87014(5)$ & & \\
\hline & $\mathrm{Mn}^{4+}{ }_{-} \mathrm{O}$ & $2.01323(5)$ & & \\
\hline & $\mathrm{Mn}^{4+}+\mathrm{O}$ & $1.92520(5)$ & & \\
\hline & $\mathrm{Mn}^{4+}-\mathrm{O}$ & $1.95823(5)$ & & \\
\hline \multirow[t]{8}{*}{ LT-O } & $\operatorname{Mn}(1)-\mathrm{O}$ & $1.88581(1)$ & $\operatorname{Mn}(1)-\mathrm{O}-\mathrm{Mn}(2)$ & $157.6742(1)$ \\
\hline & $\mathrm{Mn}(1)-\mathrm{O}$ & $1.99869(1)$ & $\operatorname{Mn}(1)-O-M n(2)$ & $162.7741(1)$ \\
\hline & $\mathrm{Mn}(1)-\mathrm{O}$ & $1.91047(1)$ & $\operatorname{Mn}(1)-O-M n(1)$ & $161.1720(1)$ \\
\hline & $\mathrm{Mn}(1)-\mathrm{O}$ & $2.01610(1)$ & $\operatorname{Mn}(2)-O-M n(2)$ & $163.4689(1)$ \\
\hline & $\mathrm{Mn}(2)-\mathrm{O}$ & $1.95475(1)$ & & \\
\hline & $\mathrm{Mn}(2)-\mathrm{O}$ & $2.01104(1)$ & & \\
\hline & $\mathrm{Mn}(2)-\mathrm{O}$ & $1.87888(1)$ & & \\
\hline & $\mathrm{Mn}(2)-\mathrm{O}$ & $2.00133(1)$ & & \\
\hline
\end{tabular}

be as isotropic as assumed in the $\mathrm{CO} / \mathrm{OO}$ state. This result anticipates a more rigorous structural analysis including a short-range order technique to give some more insight.

The third model, denoted LT-O, is the structural model used to describe the Mn-Mn dimer picture as defined by Daoud-Aladine et al $\stackrel{8}{-}$ In model LT-O the orthorhombic lattice is doubled along the $b$-axis and the atoms positions are refined with $P 2{ }_{1} n m$ symmetry constraints. Parameters from model LT-O are shown in Table IV and resulting structural information in Table $\mathrm{V}$ The third model yields a $\chi^{2}$ of 6.2 and $\mathrm{R}_{\mathrm{wp}}$ of $4.4 \%$, which are approximately equal to those of model LT-M' and slightly better than those of model LT-M. Again, the number of parameters refined in the least squares fit is approximately twice the amount in model LT-O compared to the LT-M model. Tab. $\nabla$ lists the bond lengths and angles of all the models obtained from Rietveld analysis.

Finally we have a look at the high d-spacing part of the diffraction pattern (Fig. 2). The magnetic structure was fit by an AFM phase of the CE-type using a super cell twice the size of the chemical unit cell determined at $20 \mathrm{~K}$. The symmetry of the magnetic phase is consistent with the nuclear phase. As the Shubnikov group used is $P 112_{1}^{\prime} / m$ which constrains the moments to be in the $a-b$ plane. Four different $\mathrm{Mn}$ sites were used to create the pattern and two different moments were obtained. The moment value associated to the so-called $\mathrm{Mn}^{3+}$ site in the $\mathrm{CO} / \mathrm{OO}$ model is $2.838(11) \mu_{B} / \mathrm{Mn}$ and that associated

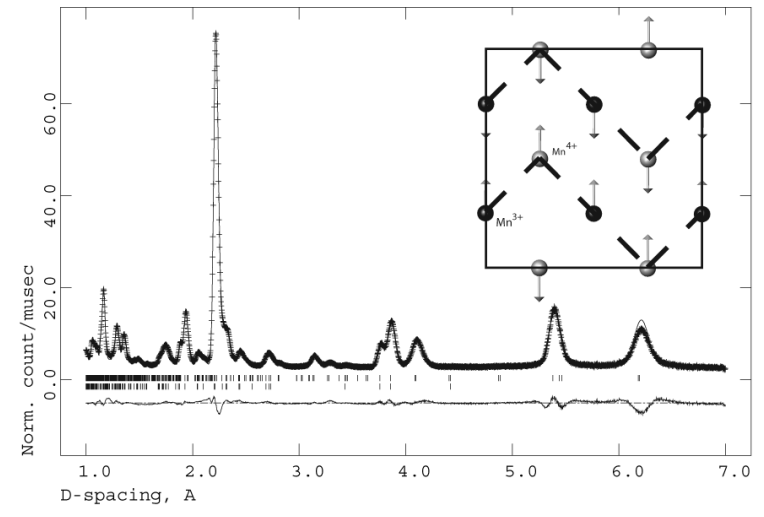

FIG. 2: NPD pattern of $20 \mathrm{~K}$ data measured on HIPD low angle detector banks. Upper tick marks indicate contribution from the CE-type AFM phase and the lower tick marks indicate the nuclear contribution as defined by model LT-M'. The inset shows the CE-type anti-ferromagnetic ordering for the $\mathrm{La}_{0.5} \mathrm{Ca}_{0.5} \mathrm{MnO}_{3}$ compound at low temperatures.

to the $\mathrm{Mn}^{4+}$ is $2.599(10) \mu_{B} / \mathrm{Mn}$.

\section{B. Pair Distribution Function Analysis}

The results from the Rietveld analysis make it obvious that analyzing the long-range order in the low temperature phase is insufficient for deciphering the subtle 
TABLE VI: Structural information from PDF analysis of models RT for $300 \mathrm{~K}$ data and LT-M' and LT-O for $20 \mathrm{~K}$ data. The angles and lengths are concerned only with the Mn to equatorial $\mathrm{O}$ bonds. $\mathrm{r}_{\max }=15 \AA$ for all the refinements.

\begin{tabular}{|c|c|c|c|c|}
\hline \multirow{2}{*}{$\begin{array}{l}\text { Model } \\
\text { RT }\end{array}$} & \multicolumn{2}{|c|}{$\mathrm{Mn}-\mathrm{O}_{e q}$ bond distances $(\AA)$} & \multicolumn{2}{|c|}{ Mn- $\mathrm{O}_{e q}$ bond angles $\left({ }^{\circ}\right)$} \\
\hline & $\mathrm{Mn}-\mathrm{O}$ & $2 \times 1.942(2)$ & Mn-O-Mn & $161.35(2)$ \\
\hline & $\mathrm{Mn}-\mathrm{O}$ & $2 \times 1.949(2)$ & & \\
\hline \multirow[t]{8}{*}{$\overline{\text { LT-M' }}$} & $\mathrm{Mn}^{3+}(1)-\mathrm{O}$ & $2 \times 1.932(4)$ & $\mathrm{Mn}^{3+}(1)-\mathrm{O}-\mathrm{Mn}^{4+}$ & $159.2(3)$ \\
\hline & $\mathrm{Mn}^{3+}(1)-\mathrm{O}$ & $2 \times 2.051(4)$ & & $166.7(3)$ \\
\hline & $\mathrm{Mn}^{3+}(2)-\mathrm{O}$ & $2 \times 1.917(4)$ & $\mathrm{Mn}^{3+}(2)-\mathrm{O}-\mathrm{Mn}^{4+}$ & $157.8(2)$ \\
\hline & $\mathrm{Mn}^{3+}(2)-\mathrm{O}$ & $2 \times 2.113(4)$ & & $161.2(2)$ \\
\hline & $\mathrm{Mn}^{4+}-\mathrm{O}$ & $1.948(6)$ & & \\
\hline & $\mathrm{Mn}^{4+}-\mathrm{O}$ & $1.845(5)$ & & \\
\hline & $\mathrm{Mn}^{4+}-\mathrm{O}$ & $1.969(6)$ & & \\
\hline & $\mathrm{Mn}^{4+}-\mathrm{O}$ & $1.884(6)$ & & \\
\hline \multirow[t]{8}{*}{$\overline{\mathrm{LT}-\mathrm{O}}$} & $\mathrm{Mn}(1)-\mathrm{O}$ & $1.89(4)$ & $\mathrm{Mn}(1)-\mathrm{O}-\mathrm{Mn}(2)$ & $161.1(4)$ \\
\hline & $\mathrm{Mn}(1)-\mathrm{O}$ & $1.901(12)$ & $\operatorname{Mn}(1)-O-M n(2)$ & $161.7(5)$ \\
\hline & $\mathrm{Mn}(1)-\mathrm{O}$ & $2.018(9)$ & $\operatorname{Mn}(1)-O-M n(1)$ & $158.2(4)$ \\
\hline & $\mathrm{Mn}(1)-\mathrm{O}$ & $1.929(10)$ & $\operatorname{Mn}(2)-\mathrm{O}-\mathrm{Mn}(2)$ & $163.5(4)$ \\
\hline & $\mathrm{Mn}(2)-\mathrm{O}$ & $2.08(5)$ & & \\
\hline & $\mathrm{Mn}(2)-\mathrm{O}$ & $1.88(3)$ & & \\
\hline & $\mathrm{Mn}(2)-\mathrm{O}$ & $2.01(3)$ & & \\
\hline & $\mathrm{Mn}(2)-\mathrm{O}$ & $1.95(3)$ & & \\
\hline
\end{tabular}

changes in the Mn octahedra ordering. Therefore, we analyzed the information hidden in the diffuse scattering of the compound through PDF analysis. The PDF can be calculated from a structural model using the relation

$$
G_{c}(r)=\frac{1}{r} \sum_{i} \sum_{j}\left[\frac{b_{i} b_{j}}{\langle b\rangle^{2}} \delta\left(r-r_{i j}\right)\right]-4 \pi r \rho_{0}
$$

where the sum goes over all pairs of atoms $i$ and $j$ within the model crystal separated by $r_{i j}$. The scattering power of atom $i$ is $b_{i}$ and $\langle b\rangle$ is the average scattering power of the sample.To account for the cutoff of $S(Q)$ at $Q_{\max }$, the calculated function $G(r)$ is then convoluted with a termination function, $\sin \left(Q_{\max } r / r\right)$. Refinements presented in this paper were carried out using the program PDFFIT ${ }^{23}$. The program allows to refine structural parameters such as lattice parameters, anisotropic atomic displacement parameters, position and site occupancies. Even though this is similar to the results of a Rietveld refinement, one needs to realize that the structural model obtained from PDF analysis is strictly only valid for length scales corresponding to the $r$-range used for the refinement. This opens up the possibility to study the local structure on different length scales by varying the $r$ range refined. In addition to structural parameters, there are two other corrections to the calculated PDF: First the finite resolution, $\Delta Q$ of the instrument leads to a dampening of the $\mathrm{PDF}$ intensities by $\exp \left(-\Delta Q^{2} r^{2} / 2\right)$. The second correction accounts for changes in the PDF peak width. The PDF peak width for a pair of atoms $i$ and $j$ is calculated $\mathrm{as}^{24}$

$$
\sigma_{i j}=\sqrt{\sigma_{i j}^{\prime 2}-\frac{\delta}{r_{i j}^{2}}-\frac{\gamma}{r_{i j}}+\alpha^{2} r_{i j}^{2}}
$$

The first term $\sigma_{i j}^{\prime}$ is the PDF peak width of the structural component due to the atomic displacement parameters. The next two parameters, $\delta$ and $\gamma$, determine the sharpening of near neighbor PDF peaks due to correlated motion, in other words the tendency to move in phase ${ }^{25.26}$ Finally the parameter $\alpha$ determines the PDF peak broadening at very high distances $r$ due to the instrument resolution. Magnetic correlations are neglected in the PDF analysis because the magnetic contribution fades quickly with increasing $Q$ compared to the nuclear contribution.

Modeling of the PDF data included two objectives: First to observe the differences between the $300 \mathrm{~K}$ and $20 \mathrm{~K}$ PDF data in order to determine how the local structure is affected and second to determine a structural model to describe the $20 \mathrm{~K}$ data applying a strategy similar to the one used in the Rietveld analysis. The PDF data obtained at $\mathrm{T}=300 \mathrm{~K}$ and $\mathrm{T}=20 \mathrm{~K}$ were fit using $\mathrm{RT}$ model over the range up to $\mathrm{r}_{\max }=15 \AA$ corresponding to the distance across $3-4 \mathrm{MnO}_{6}$ octahedra. Note that the nearest neighbor peaks in the PDF are negative because the neutron scattering length of $\mathrm{Mn}$ is negative (see Fig. 3a and b). One also observed a large peak at very low values of $r$ in the PDF which is purely due to noise propagating through the Fourier Transform. As expected, the data taken at $\mathrm{T}=300 \mathrm{~K}$ are much more accurately described by a single-cell, orthorhombic lattice than the $20 \mathrm{~K}$ data. Since the lattice parameters and atom positions are refined for both data sets, the differences seen in Fig. 3p confirm that the satellite peaks found in the NPD pattern cannot be modeled by a single cell and give rise to local structure distortions affecting the profile of the PDF peaks at $20 \mathrm{~K}$.

After comparing the room temperature and low temperature data, the low temperature data were refined 
using two different structural models, all of which used the doubled cells obtained from Rietveld analysis as initial structures. First, we used model LT-M which is the classic $\mathrm{CO} / \mathrm{OO}$ structural model constructed with space group $P 2_{1} / m$, in which only the modulation vectors $\Delta x$ and $\Delta x^{\prime}$ were refined. Again the refinement range extends to $\mathrm{r}_{\max }=15 \AA$, yielding a high $\mathrm{R}_{\mathrm{wp}}$ value of $13.4 \%$ and values of $0.0208(2)$ and $0.0165(5)$ for $\Delta x$ and $\Delta x^{\prime}$ respectively. The second step of the refinement in this model loosens the previous modulation vector constraints and allows all the atom positions to move with $P 2_{1} / m$ symmetry constraints. This second step is similar to the less constrained LT-M' structural model used in the Rietveld analysis except that the atom positions were refined after the modulation vectors $\Delta x$ and $\Delta x^{\prime}$ were applied. The second set of refinements yields a better $R_{\mathrm{wp}}$ value of $7.3 \%$ as shown in Fig. 3r and we refer to this model as LT-M' since we allow the atom positions to move with $P 2_{1} / m$ symmetry constraints.

The second model is the LT-O model, which allows the atoms to move in any direction but with $P 2{ }_{1} \mathrm{~nm}$ symmetry constraints. The result is shown in Fig. B $\mathrm{d}$. The $\mathrm{R}_{\mathrm{wp}}=12.8 \%$ indicates a much worse agreement of this model compared to the LT-M' model when refined up to $\mathrm{r}_{\max }=15 \AA$. However, if we extend the refinement range

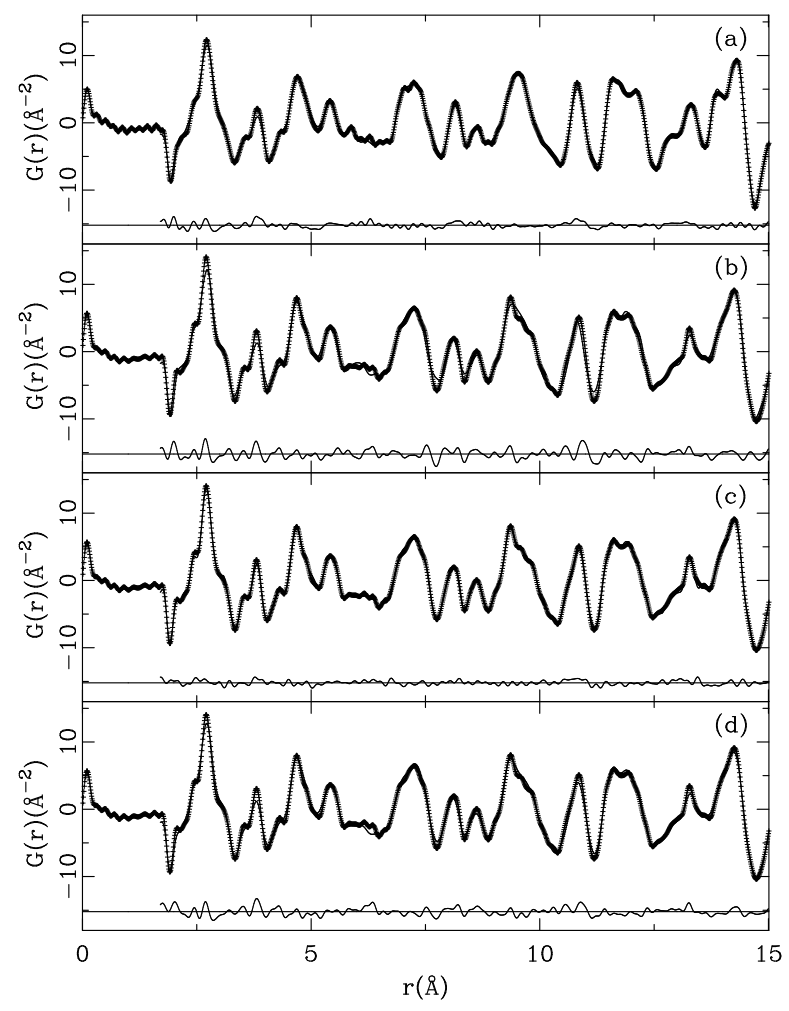

FIG. 3: PDF refinements over the range $r_{\min }=1.7 \AA$ and $\mathrm{r}_{\max }=15 \AA$ : (a) $300 \mathrm{~K}$ data using model $\mathrm{RT}, \mathrm{R}_{\mathrm{wp}}=8.8 \%$; (b) $20 \mathrm{~K}$ data using model $\mathrm{RT}, \mathrm{R}_{\mathrm{wp}}=15.2 \%$; (c) $20 \mathrm{~K}$ data using model LT-M', $\mathrm{R}_{\mathrm{wp}}=7.3 \%$ and (d) $20 \mathrm{~K}$ data using model LT-O, $\mathrm{R}_{\mathrm{wp}}=12.8 \%$. of the LT-O model to $\mathrm{r}_{\max }=45 \AA$ (approximately $12 \mathrm{Mn}$ octahedra long diameter), we then find an agreement of $\mathrm{R}_{\mathrm{wp}}=12.0 \%$ for the LT-O model which is only slightly worse compared to $\mathrm{R}_{\mathrm{wp}}=10.4 \%$ obtained for the LT-M' model. This is consistent with the Rietveld results yielding a similar agreement for both models. This means that when a PDF refinement is confined to fit only a short-range radius of $15 \AA$, it preferred the LT-M' model to the LT-O model but for longer correlations, no such preference exists. The resulting structures from the $r_{\max }$ of $15 \AA$ fit for both models are shown in Fig. 4. The LT-M' model still supports a checkerboard ordering of Mn-O octahedra. Tab. VI lists the bond lengths and angles of all the models obtained from PDF analysis for an $\mathrm{r}_{\max }=15 \AA$.

Based on the refinement of the PDF obtained at $\mathrm{T}=20 \mathrm{~K}$ up to $\mathrm{r}_{\max }=15 \AA$, model LT-M' gives the best description of the local structure of $\mathrm{La}_{0.5} \mathrm{Ca}_{0.5} \mathrm{MnO}_{3}$. However, the results of the Rietveld refinements gives no such clear distinction between the models. The power of the PDF technique is the fact that one can probe short as well as medium range order by selecting the $r$-range used in the refinement ${ }^{27}$ In order to investigate the difference between PDF and Rietveld results, we refined the $20 \mathrm{~K} \mathrm{PDF}$ data out to $\mathrm{r}_{\max }=45 \AA$ using the models LT-M' (Fig. 5 $\mathrm{p}$ ) and LT-O (Fig. 5 $\mathrm{p}$ ). The overall agreement is $\mathrm{R}_{\mathrm{wp}}=10.4 \%$ for LT-M' and $\mathrm{R}_{\mathrm{wp}}=12.0 \%$ for LT-O. However, the agreement for $15 \AA<r<45 \AA$ indicated in Fig. 5 is practically the same. This is consistent with the findings from the Rietveld refinements and we basically cannot distinguish between LT-M' and LT-O as model for the medium or long range average structure of the sample. On the other hand the local structure $(r<15 \AA)$ is quite sensitive to the differences of the two models. From the previous refinements with $r_{\max }=15 \AA$ as well as the $\mathrm{R}_{\mathrm{wp}}$-values shown for $r<15 \AA$ in Fig. [5it is obvious that locally the better structural description is model LT-M' as we have discussed above. The medium range structure obtained via PDF or the long range structure obtained via Rietveld refinements on the other hand supports both models.
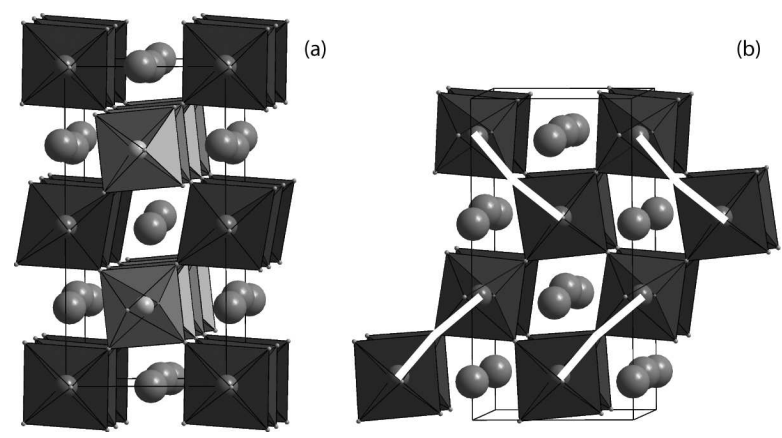

FIG. 4: (a) Resulting structure from the PDF refinement of $20 \mathrm{~K}$ data using model LT-M' for $\mathrm{r}_{\max }=15 \AA$. (b) Resulting structure from the PDF refinement of $20 \mathrm{~K}$ data using model LT-O for $\mathrm{r}_{\max }=15 \AA$. 


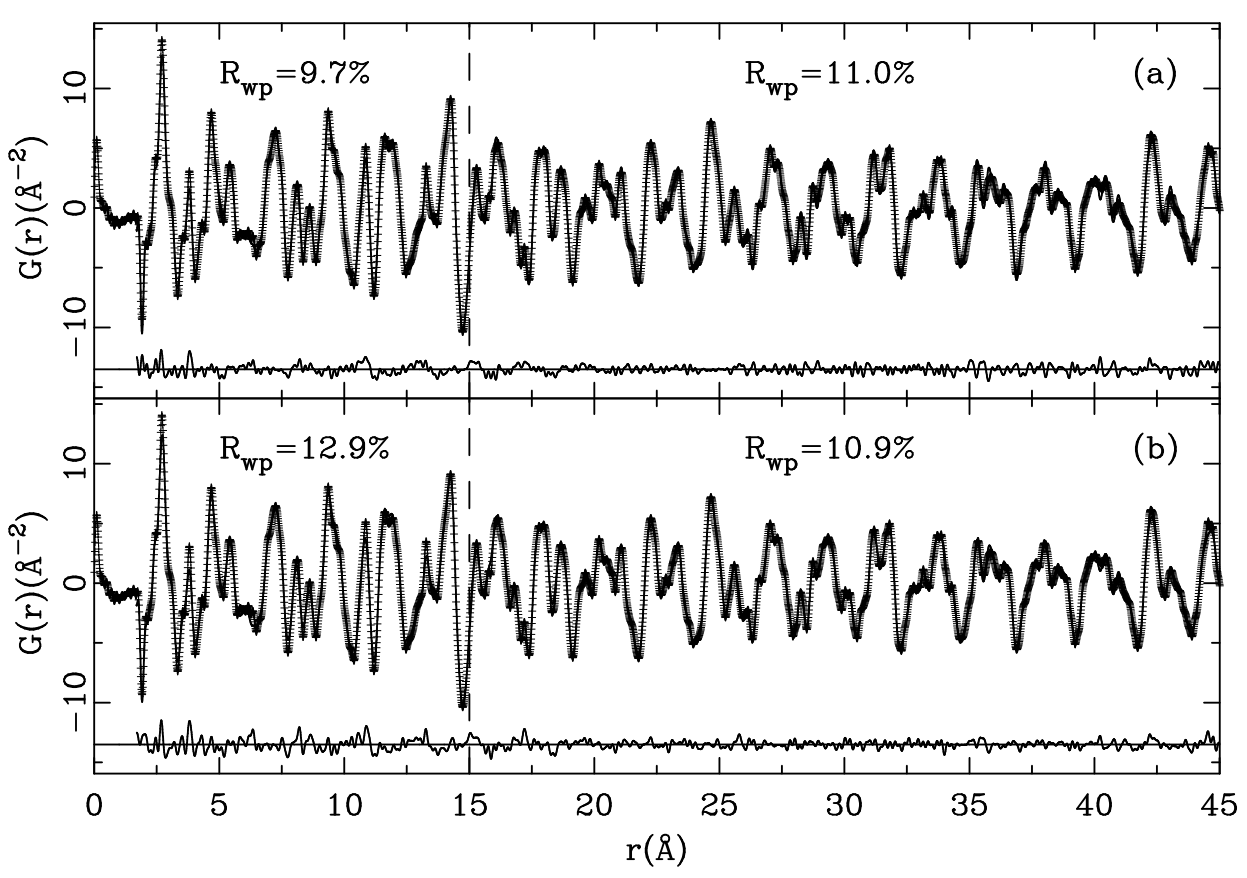

FIG. 5: PDF refinements over the range $r_{\min }=1.7 \AA$ and $r_{\max }=45 \AA$ : (a) $20 \mathrm{~K}$ data using model LT-M', $\mathrm{R}_{\mathrm{wp}}=10.4 \%$ and (b) $20 \mathrm{~K}$ data using model LT-O, $\mathrm{R}_{\mathrm{wp}}=12.0 \%$. The $\mathrm{R}_{\mathrm{wp}}$-values indicated on the panels correspond to the $\mathrm{R}_{\mathrm{wp}}$-values for the corresponding range in $r$.

\section{DISCUSSION}

One of the main criticisms about the $\mathrm{CO} / \mathrm{OO}$ model from X-ray resonance and absorption studies was that the valence around the Mn was never found to be fully $4+$ or $3+$. Indeed, our study also concludes the same about the Mn valence state as calculated from bond valence sums following the relation

$$
V_{i}=\sum_{i j} \exp \left[\frac{r_{i j}^{0}-d_{i j}}{b}\right]
$$

where $r$ and $b$ are the so-called bond valence parameters and $d_{i j}$ is the distance between the central atom $i$ and the neighboring atom $j$ 28.29 The bond valence calculations for the two models shown in Fig. 4 are presented in Tab. VII

In the LT-M' model there are $3 \mathrm{Mn}$ sites, where the $\mathrm{Mn}^{3+}(1)$ and $\mathrm{Mn}^{3+}(2)$ sites are Jahn-Teller distorted and

TABLE VII: Bond valences calculated from PDF models LTM' and LT-O refined to $\mathrm{r}_{\max }=15 \AA$. The estimated standard deviation on the last digit is given in parenthesis.

\begin{tabular}{llr}
\hline \hline Model & $M n$ site & Valence \\
\hline LT-M' & $\mathrm{Mn}^{3+}(1)$ & $3.50(4)+$ \\
& $\mathrm{Mn}^{3+}(2)$ & $3.42(4)+$ \\
& $\mathrm{Mn}^{4+}$ & $3.99(4)+$ \\
LT-O & $\mathrm{Mn}(1)$ & $3.81(4)+$ \\
& $\mathrm{Mn}(2)$ & $3.58(4)+$ \\
\hline \hline
\end{tabular}

the $\mathrm{Mn}^{4+}$ is not. It is evident that the bond valence of $\mathrm{Mn}^{3+}(1)$ and $\mathrm{Mn}^{3+}(2)$ is an appreciable amount away from the $3+$ and both close to $3.5+$ using the bond valence sums. However, the $\mathrm{Mn}^{4+}$ bond valence is actually very close to $4+$. In the LT-O model obtained from PDF analysis, there are two different $\mathrm{Mn}$ sites, which are all Jahn-Teller distorted and therefore do not correspond to $\mathrm{Mn}^{3+}$ and $\mathrm{Mn}^{4+}$ atoms. However, even in this $\mathrm{Mn}-\mathrm{Mn}$ dimer picture, there are two Mn atoms with different bond valences as shown in Table VII with one close to the $3.5+$ value and the other close to $4+$. One should keep in mind, however, that bond valence sum technique has some shortcomings in describing the bond valence states in the mixed-valence manganites.

Another result similar with the local structure analysis from X-ray data but not incompatible with the $\mathrm{CO} / \mathrm{OO}$ view is the local symmetry around the $\mathrm{Mn}^{4+}$ ion in model LT-M' obtained from PDF analysis. As demonstrated in other studies, our PDF analysis shows that the local environment around the $\mathrm{Mn}^{4+}$ atom is anisotropic as shown in Fig. 6] Instead of four equal Mn-O bond lengths on the equatorial plane of the octahedra, there are two different lengths in both directions. Therefore, the point group symmetry is lowered from a tetrahedral point symmetry of $D_{h}^{4}$ down to $C_{s}$, which only has a mirror plane. This is similar to the XANES study, where the point group symmetry of the so-called $\mathrm{Mn}^{4+}$ is not isotropic nor tetrahedral but instead monoclinic ${ }^{6}$ This suggests that the $\mathrm{Mn}^{4+}$ is slightly ferroelectric because the $\mathrm{Mn}^{4+}$ is not centered within the octahedra. However, no macroscopic polarity should be detected because the other $\mathrm{Mn}^{4+}$ in 


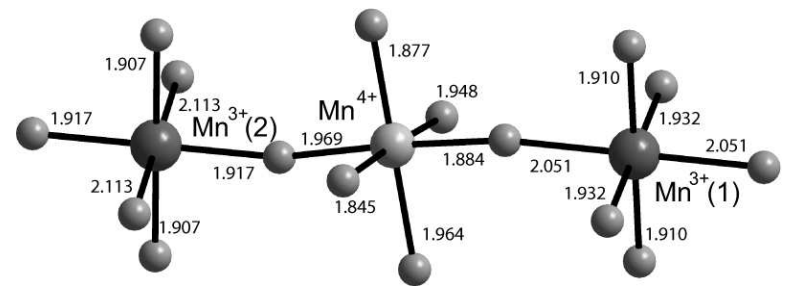

FIG. 6: Local symmetry around two Mn sites, one corresponding to the $\mathrm{Mn}^{3+}$ and $\mathrm{Mn}^{4+}$ from the LT-M' model obtained from PDF analysis. The $\mathrm{Mn}^{3+}$ octahedron is distorted in the expected Jahn-Teller way. The $\mathrm{Mn}^{4+}$ is not isotropic as expected in the $\mathrm{CO} / \mathrm{OO}$ picture but off centered.

the super-lattice is off-centered in the opposite direction and therefore should effectively cancel each other out. This is an interesting discovery concerning the $\mathrm{Mn}^{4+}$ because it demonstrates that it can be anisotropic as shown by other short-range structure studies but in a manner not contradictory to the $\mathrm{CO} / \mathrm{OO}$ picture.

Another argument against the $\mathrm{CO} / \mathrm{OO}$ picture is the similar values of the two Mn moments. In our Rietveld analysis the moment values are $2.838(11) \mu_{B} / \mathrm{Mn}$ and $2.599(10) \mu_{B} / \mathrm{Mn}$ for $\mathrm{Mn}^{3+}$ and $\mathrm{Mn}^{4+}$ respectively. Since the moment values are close, this would suggest that $e_{g}$ electron is localized over two $\mathrm{Mn}$ ions as suggested in the Mn-Mn dimer model. However, other similar studies showing Mn moments with similar magnitudes conclude that the small difference in moment values may have to do with incommensurability in the charge-ordered state, especially in the half-doped manganites $\stackrel{4.30}{ }$ One of the studies concludes that the existence of broadening of certain AFM peaks are a result of domain boundaries in $\mathrm{Mn}^{3+}$ sublattice, which causes the moment direction to flip across the magnetic domain boundary $\underline{\underline{4}}$ Therefore, the resulting incommensurability of the $\mathrm{Mn}^{3+}$ magnetic sublattice causes the refined moment value of the $\mathrm{Mn}^{3+}$ ion to average out to a lower value. Indeed, inspection of the NPD pattern in Fig. 2 shows that some of the AFM peaks are broadened more than others and would support the idea of incommensurability in our chargeordered structure.

\section{CONCLUSIONS}

Our neutron powder diffraction study on $\mathrm{La}_{0.5} \mathrm{Ca}_{0.5} \mathrm{MnO}_{3}$ has been analyzed using Rietveld and Pair distribution function analysis in order to determine the average and local structure in this controversial material. Our analysis shows that the ionic $\mathrm{CO} / \mathrm{OO}$ model and the FM dimer model succeed to equally model the low temperature charge ordered state from our powder diffraction data using Rietveld analysis (long range average structure). Therefore, a local structural analysis technique was necessary to find the coordination and bond valence state around the $\mathrm{Mn}$ ion as in X-ray local structure techniques. Our PDF analysis goes a step further than analyzing the local environment around the Mn atom (up to $4 \AA$ ). By carrying out the PDF refinements to $\mathrm{r}_{\max }=15 \AA$ and to $\mathrm{r}_{\max }=45 \AA$, we can create a structure that arranges the dissimilarly distorted Mn octahedra to bridge the gap between local structure analysis and long-range analysis from Rietveld.

The combined Rietveld/PDF analysis shows that the local structure of $\mathrm{La}_{0.5} \mathrm{Ca}_{0.5} \mathrm{MnO}_{3}$ at $20 \mathrm{~K}$ does not support the classic, strictly ionic model but does not fit the $\mathrm{Mn}-\mathrm{Mn}$ dimer structure proposed for $\mathrm{Pr}_{0.6} \mathrm{Ca}_{0.4} \mathrm{MnO}_{3}$ either. Instead, our PDF data are locally best fit by a model with $P 2_{1} / m$ symmetry (LT-M') without the constraints necessary to create the ionic, integral valence picture. This structural model supports two different Mn octahedra that are both distorted but in a different manner. One shows an anisotropic distortion associated with the Jahn-Teller effect and a bond valence state close to $3.5+$. The other has monoclinic symmetry, is clearly anisotropic, and a bond valence state close to $4+$. The anisotropy in the $\mathrm{Mn}^{4+}$ octahedra implies that the ion is slightly shifted in one direction within its octahedron. Therefore, while the system is orbital ordered, it is only partially charge ordered at low temperatures. These results reveal the importance of examining different length scales of a complex system and reveal the power of pair distribution function when coupled to Rietveld analysis.

\section{Acknowledgments}

We thank Ram Seshadri and Nicola Spaldin for helpful discussions and their insight. This work has benefited from the use of NPDF and HIPD at the Lujan Center at Los Alamos Neutron Science Center, funded by DOE Office of Basic Energy Sciences and Los Alamos National Laboratory funded by Department of Energy under contract W-7405-ENG-36. The upgrade of NPDF has been funded by NSF through grant DMR 00-76488.
* Electronic address: tproffen@lanl.gov

1 J. Coey, M. Viret, and S. V. Molnar, Adv. Phys. 48, 167 (1999).

2 E. Wollan and W. Koehler, Phys. Rev. 100, 545 (1955).

3 J. Goodenough, Phys. Rev. 100, 564 (1955).
${ }^{4}$ P. Radaelli, D. Cox, M. Marezio, and S.-W. Cheong, Phys. Rev. B 55, 3015 (1997).

5 J. Garcia, M. C. Sanchez, J. Blasco, G. Subias, and M. G. Proietti, J. Phys: Condens. Matter 13, 3243 (2001).

${ }^{6}$ G. Subias, J. Garcia, J. Blasco, M. C. Sanchez, and M. G. 
Proietti, J. Phys: Condens. Matter 14, 5017 (2002).

7 F. Bridges, C. Booth, M. Anderson, G. Kwei, J. Neumeier, J. Snyder, J. Mitchell, J. Gardner, and E. Brosha, Phys. Rev. B 63, 214405 (2001).

8 A. Daoud-Aladine, J. Rodriguez-Carvajal, L. PinsardGaudart, M. T. Fernandez-Diaz, and A. Revcolevschi, Appl. Phys. A 74, S1758 (2002).

9 J. Rodriguez-Carvajal, A. Daoud-Aladine, L. PinsardGaudart, M. T. Fernandez-Diaz, and A. Revcolevschi, Physica B 320, 1 (2002).

10 A. Daoud-Aladine, J. Rodriguez-Carvajal, L. PinsardGaudart, M. T. Fernandez-Diaz, and A. Revcolevschi, Phys. Rev. Lett. 89, 097205 (2002).

11 G. Zheng and C. Patterson, Phys. Rev. B 67, 220404 (2003).

12 J.-S. Zhou and J. Goodenough, Phys. Rev. B 62, 3834 (2000).

13 S. J. L. Billinge, R. G. DiFrancesco, G. H. Kwei, J. J. Neumeier, and J. D. Thompson, Phys. Rev. Lett. 77, 715 (1996).

14 T. Proffen, R. G. DiFrancesco, S. J. L. Billinge, E. L. Brosha, and G. H. Kwei, Phys. Rev. B 60, 9973 (1999).

15 S. J. L. Billinge, V. Petkov, T. Proffen, G. H. Kwei, J. L. Sarrao, S. D. Shastri, and S. Kycia, Mater. Res. Soc. Symp. Proc. 602, 177 (2001).

16 T. Egami and D. Louca, Phys. Rev. B 65, 094422 (2002).

17 X. Qiu, T. Proffen, J. F. Mitchell, and S. J. L. Billinge,
Phys. Rev. Lett. (2004), submitted.

18 T. Egami and S. J. L. Billinge, Underneath the BraggPeaks: Structural Analysis of Complex Materials (Elsevier Science B.V., Amsterdam, 2003).

19 T. Proffen, T. Egami, S. J. L. Billinge, A. K. Cheetham, D. Louca, and J. B. Parise, Appl. Phys. A 74, S163 (2002).

20 P. F. Peterson, M. Gutmann, T. Proffen, and S. J. L. Billinge, J. Appl. Cryst. 33, 1192 (2000).

21 A. C. Larson and R. B. Von Dreele, Report LAUR 86-748, Los Alamos National Laboratory (2000).

22 Q. Huang, J. Lynn, R. Erwin, A. Santoro, D. Dender, V. Smolyaninova, K. Ghosh, and R. Greene, Phys. Rev. B 61, 8895 (2000).

23 T. Proffen and S. J. L. Billinge, J. Appl. Cryst. 32, 572 (1999).

24 T. Proffen and S. J. L. Billinge, Manual Version 1.3, MSU (2003).

25 I. Jeong, R. H. Heffner, M. J. Graf, and S. J. L. Billinge, Phys. Rev. B 67, 104301 (2003).

${ }^{26}$ I. Jeong, T. Proffen, F. Mohiuddin-Jacobs, and S. J. L. Billinge, J. Phys. Chem. A 103, 921 (1999).

27 T. Proffen and K. L. Page, Z. Krist. 219, 130 (2004).

28 I. Brown and D. Altermatt, Acta Cryst. B41, 244 (1985).

29 N. Brese and M. O'Keeffe, Acta Cryst. B47, 192 (1991).

30 M. Dlouha, S. Vratislav, Z. Jirak, J. Hejtmanek, K. Knizek, and D. Sedmidubsky, App. Phys. A 74, S673 (2002). 